\title{
A Unified Approach to Optimal Opportunistic Spectrum Access under Collision Probability Constraint in Cognitive Radio Systems
}

\author{
Qinghai Xiao, ${ }^{1,2}$ Yunzhou Li, ${ }^{1}$ Xiaofeng Zhong, ${ }^{1}$ Xibin $\mathrm{Xu},{ }^{1}$ and Jing Wang1 \\ ${ }^{1}$ State Key Laboratory on Microwave and Digital Communications, Tsinghua National Laboratory for \\ Information Science and Technology, Department of Electronic Engineering, Tsinghua University, Beijing 100084, China \\ ${ }^{2}$ School of Electronic Technology, Information Engineering University, Zhengzhou 450004, China
}

Correspondence should be addressed to Qinghai Xiao, xiaotsinghai@gmail.com

Received 29 April 2009; Revised 15 September 2009; Accepted 18 November 2009

Academic Editor: Ying-Chang Liang

Copyright ( 2010 Qinghai Xiao et al. This is an open access article distributed under the Creative Commons Attribution License, which permits unrestricted use, distribution, and reproduction in any medium, provided the original work is properly cited.

\begin{abstract}
We consider a cognitive radio system with one primary channel and one secondary user, and then we introduce a channel-usage pattern model and a fundamental access scheme in this system. Based on this model and fundamental access scheme, we study optimal opportunistic spectrum access problem and formulate it as an optimization problem that the secondary user maximizes spectrum holes utilization under the constraint of collision tolerable level. And then we propose a unified approach to solve this optimization problem. According to the solution of the optimization problem, we analyze and present optimal opportunistic spectrum access algorithms in several cases that the idle period follows uniform distribution, exponential distribution, and Pareto or generalized Pareto distribution. Theoretical analysis and simulation results both show that the optimal opportunistic spectrum access algorithms can maximize spectrum holes utilization under the constraint that the collision probability is bounded below collision tolerable level. The impact of sensing error is also analyzed by simulation.
\end{abstract}

\section{Introduction}

Mobile and wireless communications services have experienced an explosive growth over the last decades. Increasing demand for wireless communication makes the radio spectrum more preciously. But the electromagnetic radio spectrum is a limited natural resource; the use of which is licensed by government agencies. The conventional spectrum management policies use inflexible spectrum assignment to prevent mutual interference all the time. This has led to the artificial radio spectrum scarcity that most of the available radio spectrum has already been allocated to various services. The frequency allocation chart [1] in the United States indicates multiple allocations over all of the frequency bands. On the other hand, careful studies of the spectrum usage pattern by Spectrum Policy Task Force (SPTF) have revealed that many portions of the allocated radio spectrum experience low utilization and they are either unoccupied or partially occupied for long periods of time [2]. In fact, recent measurements have shown that $70 \%$ of the allocated spectrum is not utilized [2]. Extensive measurements also indicate that many portions of licensed spectrum lie unused at any given time and location [3]. Even when a channel is actively used, the bursty arrivals of many applications result in abundant spectrum opportunities at the slot level.

Growing demand and low utilization for the radio spectrum motivate the concept of spectrum reuse, which forms the key rationale for opportunistic spectrum access (OSA) coined by the DARPA XG program [4]. The OSA system requires that the secondary user efficiently utilizes unoccupied spectrum holes while avoiding interference with primary users [5]. The spectrum usage patterns of primary users vary over time. Thus, the secondary user experiences dynamic spectrum holes and needs to intelligently adapt its channel usage. In conventional methods, the secondary 
user senses local channels through individual or cooperative sensing [6-10] and reconfigures its access parameters according to the channel-usage patterns of primary users. This adaptation is based on the current observation of the spectrum usage by primary users. Once detecting a primary user's occurrence on its current band in use, the secondary user pauses transmissions, starts to sense the channel, and awaits next opportunity to resume transmissions. The conventional methods cannot schedule future transmissions without any prior information about future spectrum holes and result in that the secondary user frequently collides with primary users. Collisions occur when the secondary user cannot predict the appearance of primary users and can only react to current observations of primary users. In this paper, we propose an OSA approach based on spectrum holes prediction where the secondary user builds a predictive model of primary users' channel usage and estimates future spectrum holes based on past observations.

There have been several prior works on dynamic spectrum access and sensing. The most relevant works are [1114]. In [11], the authors proposed a proactive access scheme based on the characteristics of TV broadcast and explored the feasibility of proactive access method. In [12], the authors extended this work to the exponential ON-OFF model. Our work discusses OSA problems based on spectrum holes prediction while primary user traffic model is general model. Moreover, [11] mainly focuses on maximizing throughput of the secondary user, and [12] mainly focuses on minimizing disruptions to primary users, while our work focuses on maximizing spectrum holes utilization on the basis of satisfying the constraint of collision tolerable level allowed by primary network. In $[13,14]$, the authors study the optimal design of the transmission time in one collision case that collision occurs since the secondary user performs imperfect sensing, but they both do not consider the other collision case that collision occurs since the primary user reoccurs when the secondary user is transmitting. In our work, we assume that the secondary user performs perfect sensing and study the optimal design of the transmission time in the latter collision case.

Our last work [15] has investigated the optimal design of the transmission time in the case that the idle period follows exponential distribution and presented an optimal OSA approach to maximize spectrum holes utilization under the constraint of collision tolerable level in this case. In this work, we propose a unified approach to optimal OSA approach under the constraint of collision tolerable level in more general cases.

The remainder of this paper is organized as follows. The next section describes the system model and fundamental access scheme. The relevant concepts of channel utilization and collision probability are explained in Section 3. The optimization problem is formulated and a unified approach to optimal OSA is proposed in Section 4. Several cases that the idle period is uniform distribution, exponential distribution, and generalized Pareto distribution are analyzed in Section 5. Corresponding simulation and numerical results are presented in Section 6. Our main conclusions are summarized in the final section.

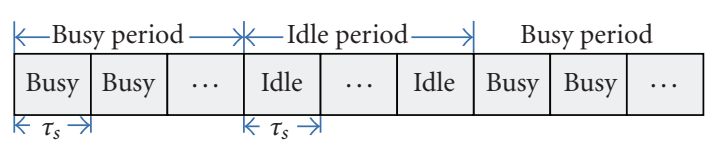

Figure 1: Channel-usage pattern model.

\section{System Model}

In this section, we consider the channel-usage pattern model in the system with one primary channel and one secondary user and propose a fundamental access scheme.

2.1. Channel-Usage Pattern Model. Consider a system with one primary channel and one secondary user. Primary users are the licensed users of this channel and thus have higher priority over the secondary user. The channel is called idle if it is unoccupied by one or more primary users and is busy otherwise (Figure 1). The duration of idle period is the time interval starting at the release of the channel until the first packet arrival. Similarly, the duration of busy period is the time interval starting at the first packet arrival until the moment that the channel becomes idle. The primary system does not employ slotted protocol and the primary users can access primary channel at any time, while the secondary user system adopts a slotted communication in spite of the primary user system.

In this study, for the convenience of analysis, we assume that (i) the system is stationary and ergodic, (ii) the secondary user performs perfect sensing at the beginning of every time slot, that is, both false alarm and missing probability are zero, and (iii) the sensing time is much less than the duration of time slot and the sensing time can be ignored. We mainly study how to obtain optimal OSA approach in the case that the idle period follows different distribution. Moreover, we will also analyze the impact of sensing errors by simulation.

2.2. Fundamental Access Scheme. In this study, the secondary user employs the following fundamental access scheme.

(1) Keep silent if busy. The secondary user keeps silent if it senses the channel busy.

(2) Keep silent and transmit in turn if idle. The secondary user can adopt a time allocation strategy of the idle period to decide whether to keep silent or transmit in current time slot if it senses the channel idle.

On the basis of fundamental access scheme, we will study optimal time allocation strategy of the idle period and compare the performance of optimal strategy and other strategies.

\section{Channel Utilization and Collision Probability}

3.1. Channel Utilization and Spectrum Holes Utilization. Channel utilization (CU) of the primary users is defined as the fraction of time in which the channel is occupied by the primary users, that is, the channel is in ON (busy) 


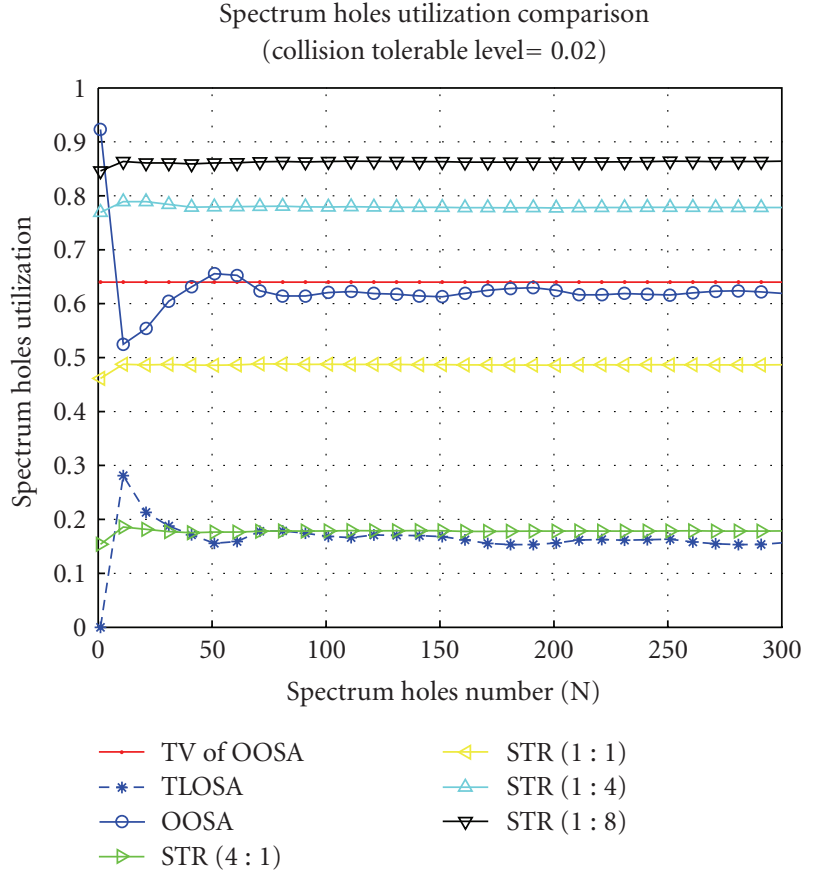

(a)

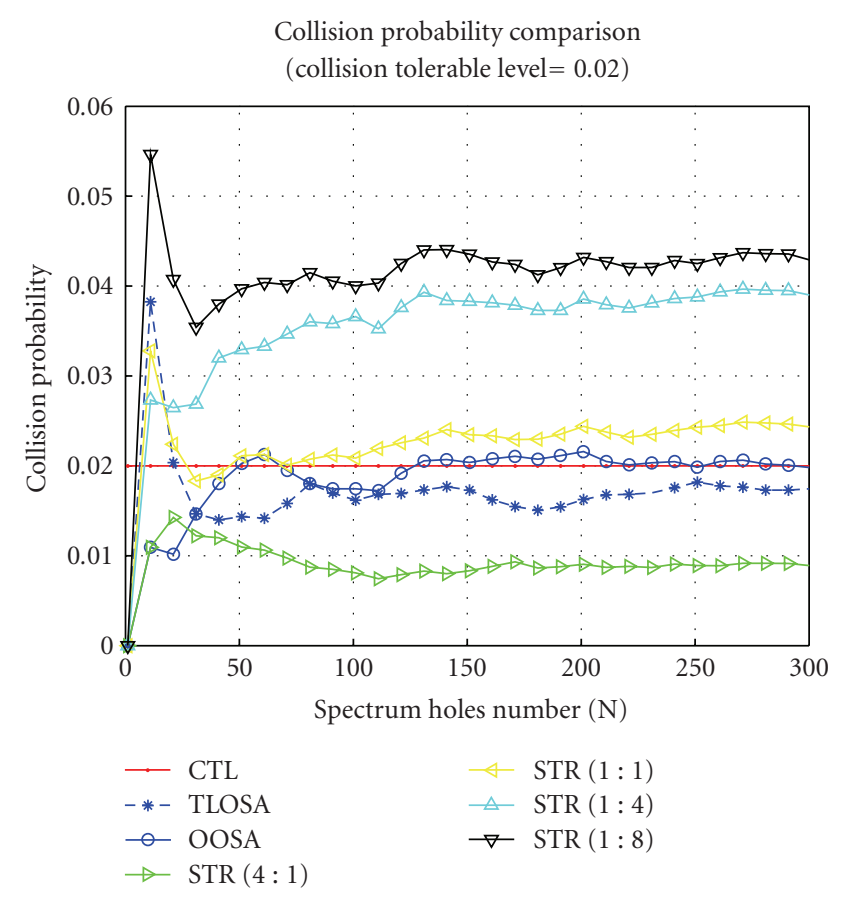

(b)

FIGURE 2: Performance comparison between optimal OSA approach and fixed STR method (collision tolerable level $\varsigma=0.02$ ) in the case that the idle period follows uniform distribution.

state, denoted by $\eta_{\mathrm{PU}}$. Under assumption of stationarity and ergodicity, it can be given as [16]

$$
\eta_{\mathrm{PU}}=\lim _{T \rightarrow \infty} \frac{\text { Duration of busy time slots of PU in }[0, T]}{T} .
$$

Channel utilization of the secondary user is defined as the fraction of time in which the channel is utilized by the secondary user, denoted by $\eta_{\mathrm{su}}$.

The definition of spectrum hole is given in [17]. In this paper, we only concern spectrum holes of one primary channel. We define spectrum holes utilization of the channel as

$\eta_{\mathrm{SH}}$

$=\lim _{T \rightarrow \infty} \frac{\text { Duration of spectrum holes utilized by SU in }[0, T]}{\text { Duration of all spectrum holes in }[0, T]}$.

Obviously, we can obtain that channel utilization of the secondary user is

$$
\eta_{\mathrm{SU}}=\left(1-\eta_{\mathrm{PU}}\right) \eta_{\mathrm{SH}} .
$$

Therefore, after the secondary user accesses the channel, the aggregate channel utilization of the channel can be given as

$$
\eta=\eta_{\mathrm{PU}}+\eta_{\mathrm{SU}}=\eta_{\mathrm{PU}}+\left(1-\eta_{\mathrm{PU}}\right) \eta_{\mathrm{SH}} .
$$

According to (4), we can see that the aggregate channel utilization $\eta$ increases linearly with spectrum holes utilization
$\eta_{\mathrm{SH}}$ when $\eta_{\mathrm{PU}}$ is certain. That is to say, optimizing aggregate channel utilization $\eta$ is the equivalent of optimizing spectrum holes utilization $\eta_{S H}$ if the channel usage of the primary users is certain.

3.2. Collision Probability. Because the secondary user performs perfect sensing, collisions happen only when primary users reoccur and occupy the channel while the secondary user is transmitting. Collision probability (CP) is the probability of the secondary transmission colliding with the primary transmission. In this study, we assume that the secondary user transmits failed completely if a collision occurs in a time slot. Thus, under the assumption of stationarity and ergodicity, we can define collision probability as

$$
p^{c}=\lim _{T \rightarrow \infty} \frac{\text { Number of collision time slots in }[0, T]}{\text { Number of busy time slots of PU in }[0, T]} .
$$

3.3. Collision Tolerable Level. In cognitive radio network, though the secondary user can be allowed to utilize the idle spectrum unoccupied by primary users, the collision probability of the primary users should be less than a threshold [18]. Collision tolerable level (CTL) is defined as the maximum probability of collision allowed by the primary users, denoted by $\varsigma$. The wireless communication systems, which provide with different services in different networks, can tolerate different collision types and collision probability. For example, voice service is real time but it can tolerate a few packet loss rate. Whereas, data service cannot lose packet but it may tolerate a little time delay. Therefore, almost all of 


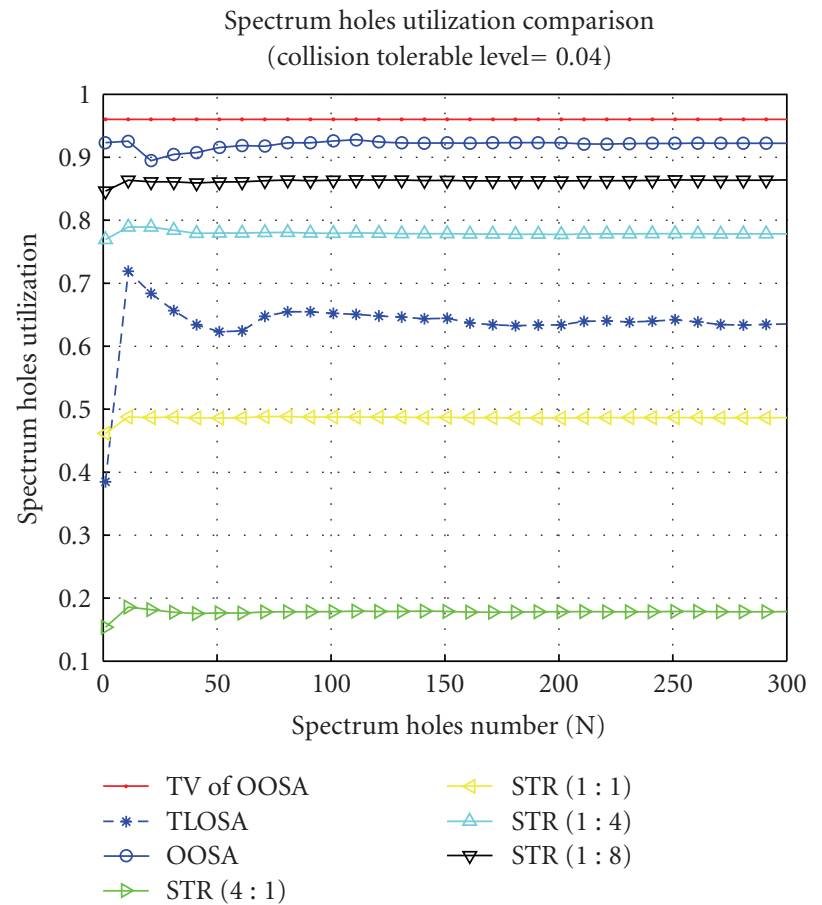

(a)

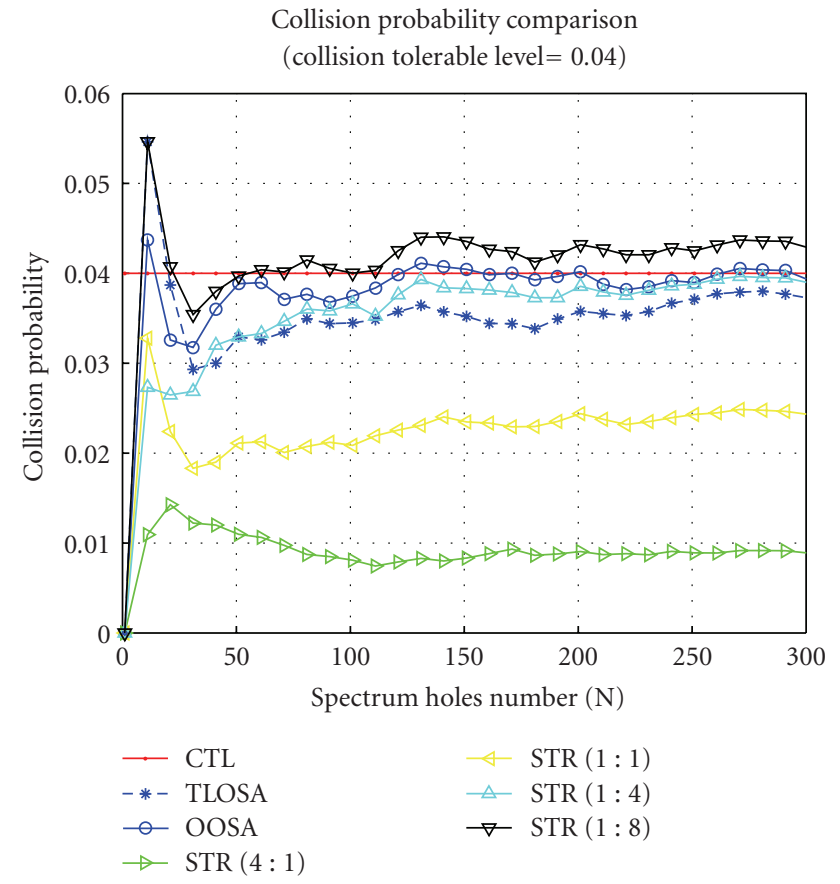

(b)

FIGURE 3: Performance comparison between optimal OSA approach and fixed STR approach (collision tolerable level $\varsigma=0.04$ ) in the case that the idle period follows uniform distribution.

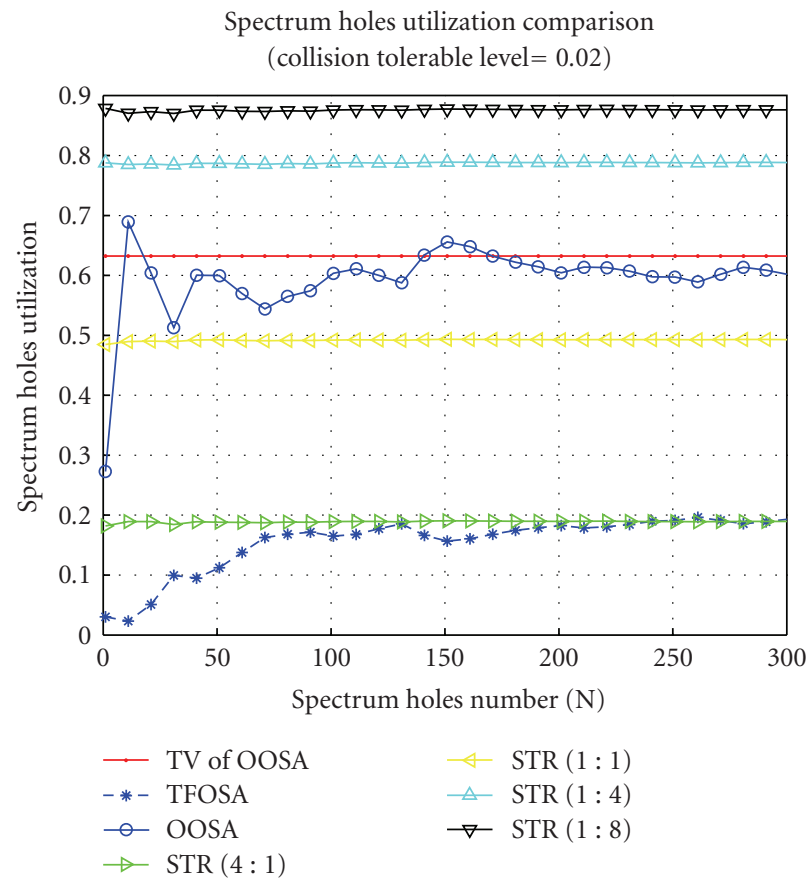

(a)

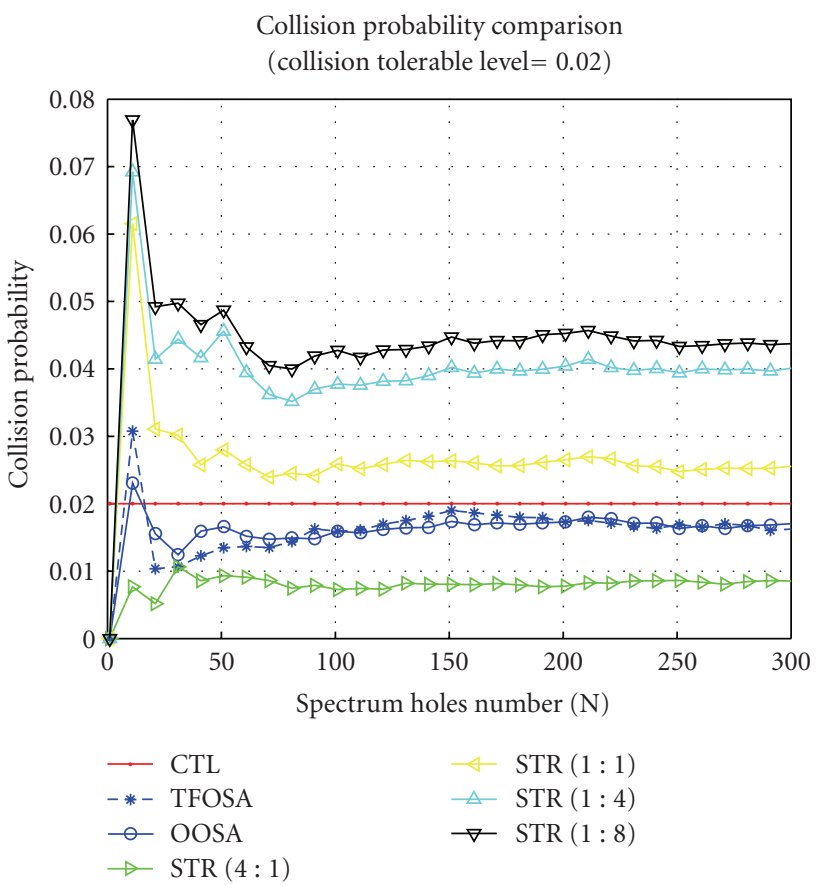

(b)

FIGURE 4: Performance comparison among optimal OSA approach and transmission-first OSA approach and fixed STR approach (collision tolerable level $\varsigma=0.02$ ) in the case that the idle period follows general Pareto distribution. 


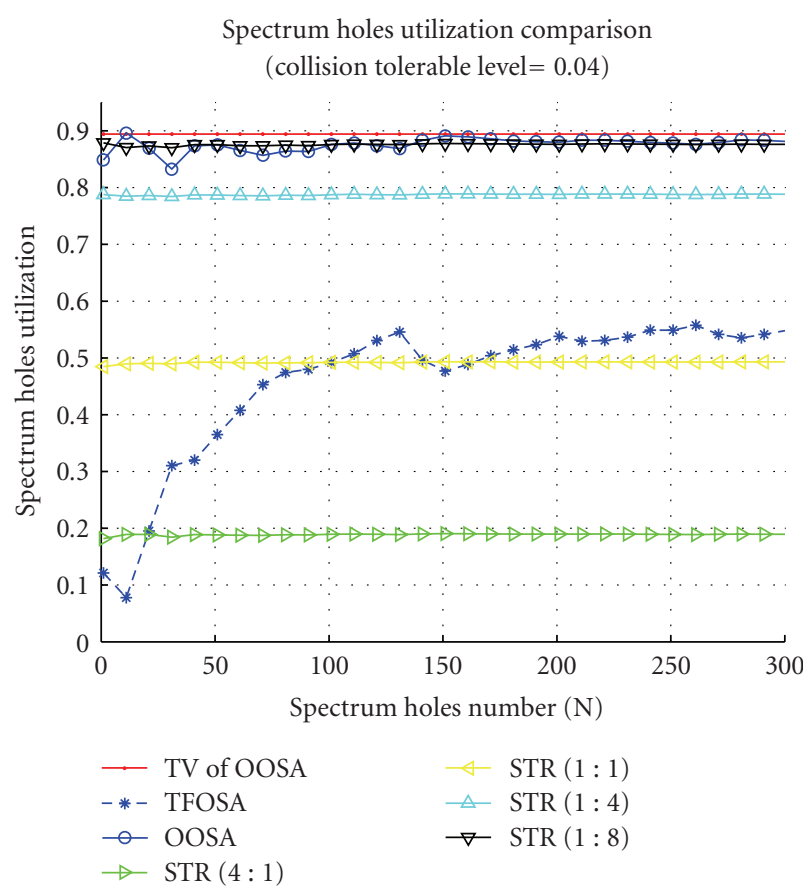

(a)

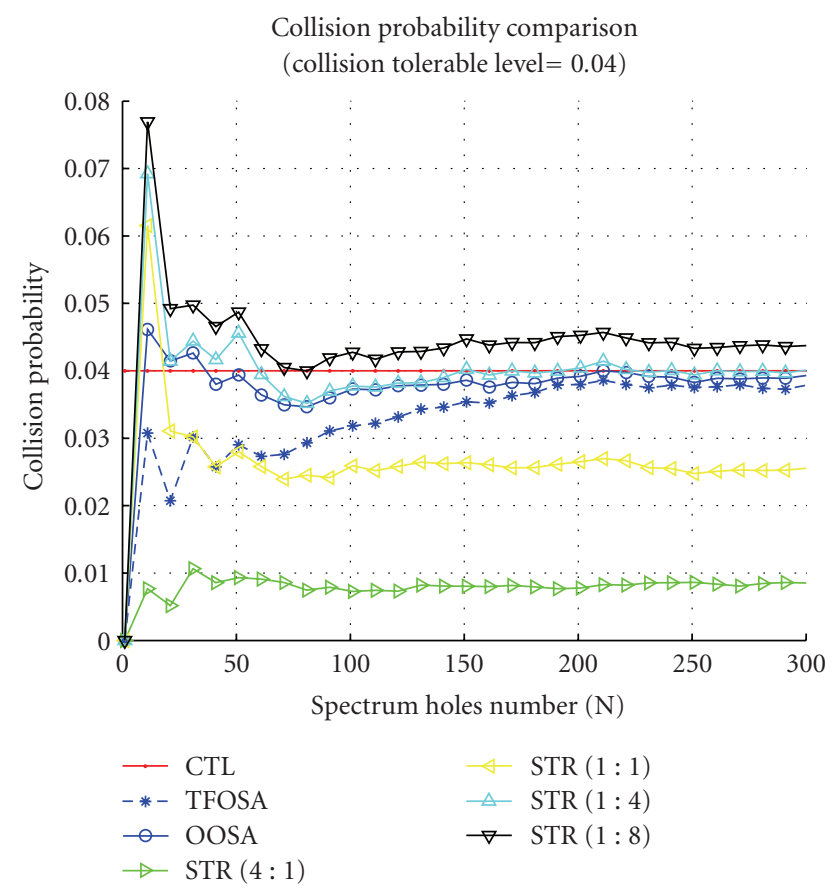

(b)

FIGURE 5: Performance comparison among optimal OSA approach and transmission-first OSA approach and fixed STR approach (collision tolerable level $\varsigma=0.04$ ) in the case that the idle period follows general Pareto distribution.

different services can tolerate a few collisions despite of the difference of collision types. In our work, we do not place emphasis on studying the differences of collision types, but we only assume that the primary users can accept collision tolerable level $\varsigma$. Collision tolerable level is also collision probability constraint of the cognitive radio system. Thus, the system must satisfy

$$
0 \leq p^{c} \leq \varsigma
$$

Otherwise, too many collisions will affect the primary users' transmission.

3.4. Identifying Collision. Due to performing perfect sensing, collisions occur only when primary users reoccur and occupy the channel while the secondary user is transmitting. Because the secondary user senses the channel at the beginning of every time slot, it can but regard this case as collision that it transmits in previous time slot and it senses the channel busy in current time slot. Though there exists this case that the primary users start transmitting at the time of the secondary user starting sensing, these do not increase collision probability.

3.5. Maximum Collision Probability. Because the secondary user can exactly sense the channel at the beginning of every time slot, we can understand that there exists at most one collision slot at the beginning of every busy period. And in the fundamental access scheme, the secondary user adopts this strategy that it keeps transmitting if it senses the channel idle in every time slot. Obviously, the access strategy has the maximum collision probability (MCP), denoted by $P_{\max }^{c}$. Under the assumption of stationarity and ergodicity, we can obtain the following expression on average:

$$
P_{\max }^{c}=\lim _{N \rightarrow \infty} \frac{N}{\sum_{i=1}^{N} 1 / v_{i}}=\lim _{N \rightarrow \infty} \frac{N}{N / v}=v,
$$

where $1 / v_{i}$ is the duration of the $i$ th busy period of the channel. We can see from (7) that the maximum collision probability is equal to the reciprocal of the average value of the busy period.

3.6. Fixed STR Approach. On the basis of the fundamental access scheme, an intuitive time allocation strategy of the idle period is periodic sensing and accessing strategy. We refer to this strategy as fixed silence duration and transmission duration ratio (STR) approach. In fixed STR approach, time allocation strategy is that the secondary user keeps silent and transmits for fixed integral-number time slots in turn if it senses the channel idle in every time slot. That is to say, once sensing the channel idle in every time slot, the secondary user keeps silent for fixed $D$ time slots and then starts to transmit and keeps transmitting for fixed $T$ time slots in turn until the secondary user senses the channel busy.

However, the fixed STR approach does not consider the joint design of spectrum holes utilization and collision probability and it results in uncontrollable collision probability. Thus, it cannot optimize spectrum holes utilization under 


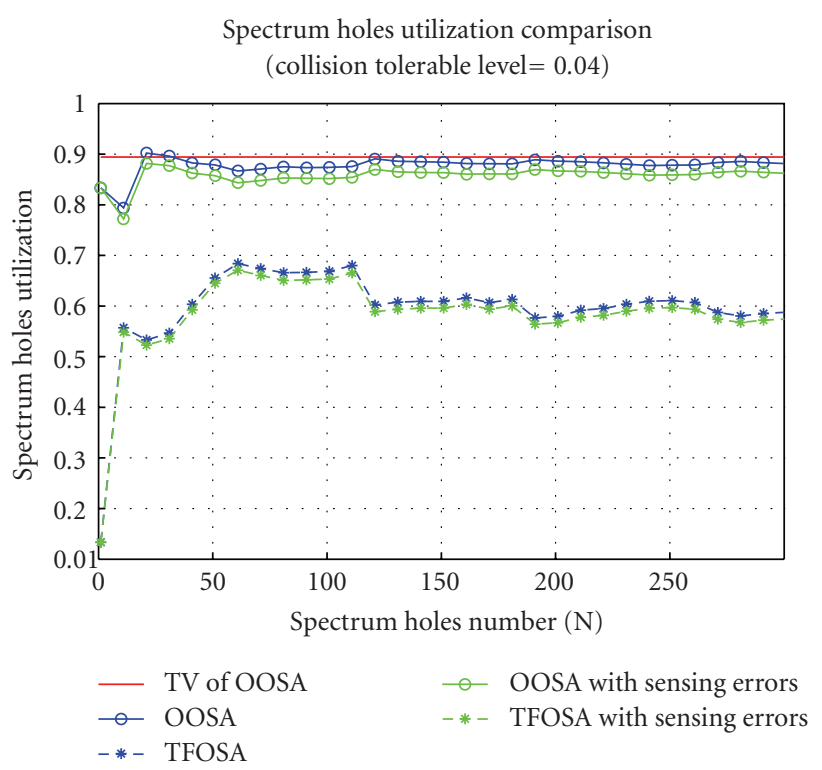

(a)

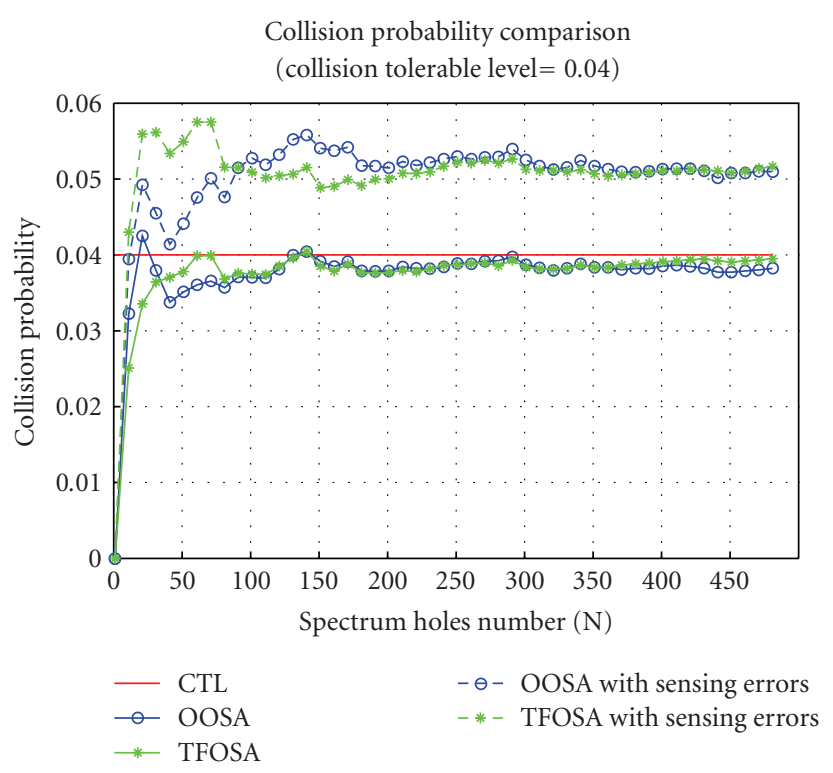

(b)

FIGURE 6: Robustness comparison between optimal OSA approach and transmission-first OSA approach (collision tolerable level $\varsigma=0.04$ and probability of sensing error is 0.02 ) in the case that the idle period follows general Pareto distribution.

Spectrum holes utilization comparison (collision tolerable level $=0.04$ )

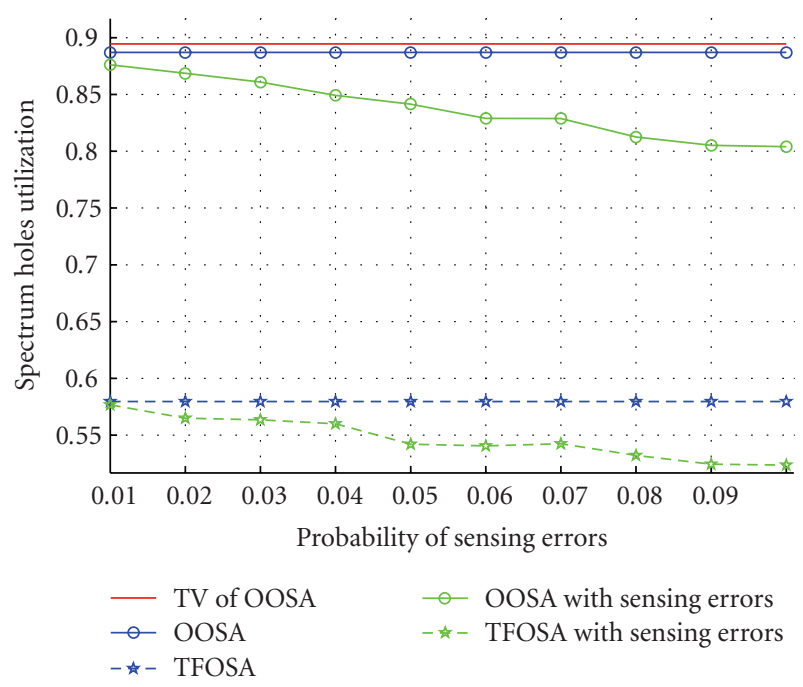

(a)

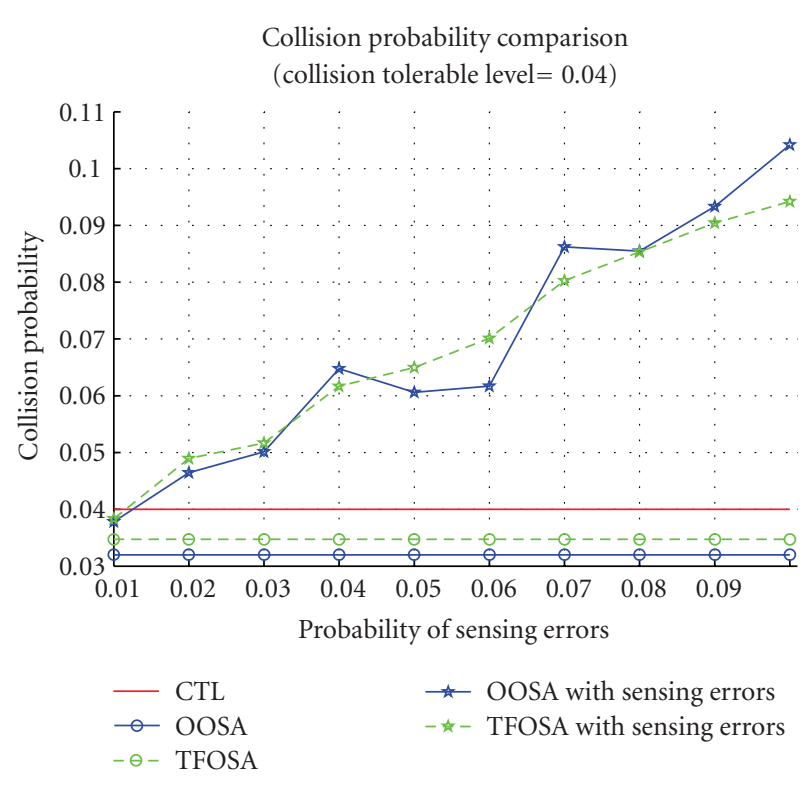

(b)

FIGURE 7: Robustness comparison between optimal OSA approach and transmission-first OSA approach (collision tolerable level $\varsigma=0.04$ ) in the case that the idle period follows general Pareto distribution.

the constraint of collision tolerable level, and it cannot also adapt its access parameters in accordance with the change of environment, such as various collision tolerable level, various channel-usage pattern, and so forth.

To solve this problem, we will propose an optimal OSA approach where the secondary user adapts its access parameters based on channel-usage estimate in the next several sections. Our aim is to maximize the spectrum holes utilization under the constraint of collision tolerable level.

\section{Unified Approach to Optimal OSA}

In this section, we propose a unified approach to optimal opportunistic spectrum access where the secondary user adapts its access parameters based on channel-usage estimate in different cases that the idle period follows different probability distribution. Our objective is to maximize the spectrum holes utilization under the constraint of collision tolerable level. 
4.1. Problem Formulation. In optimal OSA approach, we consider that the secondary user at most accesses the channel one time in an idle period. The secondary user starts to transmit at the $x$ th time slot of the idle period and keeps transmitting $T(x)$ time slots, where $T(x)$ is a function of $x$. We denote $T(x)$ as $T$ for convenience. Intuitively, the secondary user should immediately access the channel after sensing the channel idle, that is, $x$ should always be zero. However, when the secondary user maximizes the spectrum holes utilization under the constraint of collision tolerable level, it is possible that $x$ be a positive value. And in fact, we will also prove and verify by simulation that $x$ is greater than zero when the idle period follows generalized Pareto distribution (see Section 5.3).

Now our optimization problem is to maximize spectrum holes utilization under the constraint of collision tolerable level by selecting one time interval for transmitting in the idle period. Under the assumption of stationarity and ergodicity, transmission duration expectation can be given as

$$
E(x, T)=\int_{x}^{x+T}(t-x) f(t) d t+T \int_{x+T}^{\infty} f(t) d t,
$$

where $f(t)$ is the probability density function of the idle period. The first part of the right side of (8) represents the transmission duration expectation that the idle period terminates in the time interval $[x, x+T)$ and the second part of the right side of (8) represents the transmission duration expectation that the idle period does not terminate in the time interval $[x, x+T)$.

From (8), we can obtain

$$
E(0, \infty)=\int_{0}^{\infty} t f(t) d t=E(t)
$$

where $E(t)$ is the expectation value of the idle period.

According to the definition of spectrum holes utilization, we can formulate the spectrum holes utilization as the following expresses that

$$
\eta_{\mathrm{SH}}=\frac{E(x, T)}{E(0, \infty)}
$$

On the other hand, we can formulate the collision probability as the following expresses that

$$
P^{c}=P^{c}(x, T)=P_{\max }^{C} \int_{x}^{x+T} f(t) d t .
$$

We are now ready to formally state the optimization problem as follows.

Given that the distribution of the idle period is fixed, maximize the spectrum holes utilization, subject to the constraint that the collision probability is bounded below collision tolerable level. as

That is to say, we can formulate the optimization problem

$$
\begin{aligned}
& \max _{x} \eta_{\mathrm{SH}}, \\
& \text { s.t. } \quad P^{c} \leq \varsigma .
\end{aligned}
$$

According to (8), (9), (10), (11), and (12) we can obtain the optimization problem as follows:

$$
\begin{aligned}
& \max _{x}\left\{\frac{\int_{x}^{x+T}(t-x) f(t) d t+T \int_{x+T}^{\infty} f(t) d t}{\int_{0}^{\infty} t f(t) d t}\right\}, \\
& \text { s.t. } \quad P_{\max }^{C} \int_{x}^{x+T} f(t) d t \leq \varsigma .
\end{aligned}
$$

4.2. Optimal OSA Approach. It is easily understood that the secondary user should have optimal access time slot and available transmission duration in the idle period under the constraint of collision tolerable level, denoted by $x^{\mathrm{opt}}$ and $T^{a}$, respectively. In this subsection, we discuss how to obtain $x^{\text {opt }}$ and $T^{a}$ in the following two cases.

Case 1 ( $\left.\varsigma \geq P_{\max }^{c}\right)$. From Section 3, we know that collision probability $P^{c}$ must be less than or equal to maximum collision probability $P_{\max }^{c}$. Thus, in spite of transmission duration, collision probability $P^{c}$ must also be less than or equal to collision tolerable level $\varsigma$. Therefore, the secondary user can start to transmit at the first time slot in the idle period and keep transmitting until collision occurs. That is to say, available transmission duration is limitless, that is,

$$
x^{\mathrm{opt}}=0, \quad T^{a}=\infty .
$$

Thus, (14) is always true when $\varsigma \geq P_{\max }^{c}$.

Case $2\left(\varsigma<P_{\max }^{c}\right)$. We can see from $(13)$ that $E(0, \infty)$ is certain and constant if the idle period distribution is certain. Thus, maximizing spectrum holes utilization $\eta_{\mathrm{SH}}$ is the equivalent of maximizing transmission duration expectation $E(x, T)$. This point will be used in the proof of the following theorem.

Theorem 1. Assume that $x_{\max }^{\mathrm{opt}}$ satisfies

$$
\begin{aligned}
& \int_{x_{\max }^{\mathrm{opt}}}^{\infty} f(t) d t=\frac{\varsigma}{v}, \\
& g(x)=\frac{\int_{x}^{\infty} f(t) d t}{f(x)} .
\end{aligned}
$$

In optimization problem $(13)$, in the case that $\varsigma<P_{\max }^{c}$, the following conclusions can be obtained.

(1) If $g(x)$ is monotonically decreasing with $x$, then the optimal access time slot is

$$
x^{\mathrm{opt}}=0,
$$

and available transmission duration $T^{a}$ satisfies

$$
\int_{0}^{T^{a}} f(t) d t=\frac{\varsigma}{v} .
$$

(2) If $g(x)$ is constant, then the optimal access time slot is

$$
x^{\mathrm{opt}}= \begin{cases}\text { arbitrary in }\left[0, x_{\max }^{\mathrm{opt}}\right], & \varsigma<P_{\max }^{c}, \\ 0, & \varsigma \geq P_{\max }^{c},\end{cases}
$$


and available transmission duration $T^{a}$ satisfies

$$
\int_{x^{\mathrm{opt}}}^{x^{\mathrm{opt}}+T^{a}} f(t) d t=\frac{\varsigma}{v} .
$$

(3) If $g(x)$ is monotonically increasing with $x$, then the optimal access time slot is

$$
\begin{gathered}
x^{\mathrm{opt}}= \begin{cases}x_{\max }^{\mathrm{opt}}, & \varsigma<P_{\max }^{c}, \\
0, & \varsigma \geq P_{\max }^{c},\end{cases} \\
T^{a}=\infty
\end{gathered}
$$

(4)

$$
\eta_{\mathrm{SH}, \max }=\frac{E\left(x^{\mathrm{opt}}, T^{a}\right)}{E(0, \infty)} .
$$

Proof. See Appendix A.

\section{Case Analysis}

In this section, we study several practical cases that the idle period follows uniform distribution, exponential distribution, and Pareto or generalized Pareto distribution, deduce several corollaries of Theorem 1 in these cases, and present optimal OSA algorithm according to these corollaries.

5.1. Uniform Distribution. In this subsection, we solve the optimization problem (13) in the simplest case that the idle period is uniform distribution.

We assume that the idle period is uniform distribution and its expectation is $a / 2$ while the average value of busy period is $1 / v$. Thus, the idle period $X$ is uniform distribution with probability density function

$$
f(x)= \begin{cases}\frac{1}{a} & \text { for } 0 \leq x \leq a, \\ 0 & \text { for } x<0 \text { or } x>a .\end{cases}
$$

Corollary 2. If the idle period is uniform distribution, then the solution of optimization problem (13) is that optimal access time slot is

$$
x^{\mathrm{opt}}=0
$$

available transmission duration $T^{a}$ is

$$
T^{a}= \begin{cases}\frac{a \varsigma}{v}, & \varsigma<P_{\max }^{c}, \\ \infty, & \varsigma \geq P_{\max }^{c},\end{cases}
$$

and maximum spectrum holes utilization is

$$
\eta_{\mathrm{SH}, \max }= \begin{cases}\frac{2 \varsigma}{v}-\frac{\varsigma^{2}}{v^{2}}, & \varsigma<P_{\max }^{c}, \\ 1, & \varsigma \geq P_{\max }^{c} .\end{cases}
$$

Proof. See Appendix B.

We can see from Corollary 2 that in the case that the idle period is uniform distribution the optimal OSA approach is that the secondary user starts transmission at the 1st time slot after sensing the channel idle.
5.2. Exponential Distribution. In this subsection, we solve the optimization problem (13) in the case that the idle period is exponential distribution.

We assume that the arrival process of one primary user is Poisson process while the service time distribution can be arbitrary. This assumption holds in many situations such as voice traffic, data session, and data network. When there are multiple primary users in a channel, the system can be modeled as an M/G/1 queue with multiple inputs and it can be proved that the idle period is exponential distribution while the busy period is general distribution [8]. Thus, we can assume that the idle period is exponential distribution and its expectation is $1 / u$ while the busy period is general distribution and its average value is $1 / v$. Thus, the idle period $X$ is exponential distribution with probability density function

$$
f(t)=u e^{-u x} \text { for } x \geq 0 .
$$

Corollary 3. If the idle period is exponential distribution, then the solution of optimization problem (13) is that optimal access time slot is

$$
x^{\mathrm{opt}}= \begin{cases}\text { arbitrary in }\left[0, \frac{1}{u \ln (v / \varsigma)}\right], & \varsigma<P_{\max }^{c}, \\ 0, & \varsigma \geq P_{\max }^{c},\end{cases}
$$

available transmission duration is

$$
T^{a}= \begin{cases}-\frac{\ln \left(1-\varsigma e^{\left.u x^{\mathrm{opt}} / v\right)}\right.}{u}, & \varsigma<P_{\max }^{c}, \\ \infty, & \varsigma \geq P_{\max }^{c}\end{cases}
$$

and maximum spectrum holes utilization is

$$
\eta_{\mathrm{SH}, \max }= \begin{cases}\frac{\varsigma}{v}, & \varsigma<P_{\max }^{c}, \\ 1, & \varsigma \geq P_{\max }^{c} .\end{cases}
$$

Proof. See Appendix C.

We can see from Corollary 3 that the optimal OSA approach is that the secondary user starts to transmit at an arbitrary time slot in $\left[0, x_{\max }^{\mathrm{opt}}\right]$, where $x_{\max }^{\mathrm{opt}}=0$ or $1 / u \ln (v / \varsigma)$ and keeps transmitting for $T^{a}=\infty$ or $-\ln \left(1-\varsigma e^{u x} / v\right) / u$, respectively. This result is identical to [15].

5.3. Generalized Pareto Distribution. In this section, we solve the optimization problem (13) in the case that the idle period is generalized Pareto distribution.

Research [19] shows that an exponential distribution is a good fit for the idle period only in heavy traffic case while a generalized Pareto distribution is a good fit for the idle period in both heavy-traffic and small-traffic cases. Thus, in this section, we extend our work to more general case that the idle period is Pareto distribution or generalized Pareto distribution while the busy period still is general distribution and its average value is $1 / v$. Thus, we consider 
that the duration of the idle period $X$ is generalized Pareto distribution with probability density function [20]

$$
f(x ; k, \sigma)=\frac{1}{\sigma}\left(1+k \frac{x}{\sigma}\right)^{-1-1 / k},
$$

where $k \neq 0$ is the shape parameter, and $\sigma$ is the scale parameter. It should be noted that for $k=0$ the generalized Pareto distribution converges to the exponential distribution.

Corollary 4. Given that the idle period is generalized Pareto distribution, the solution of optimization problem (14) is that optimal access time slot is

$$
x^{\mathrm{opt}}= \begin{cases}\frac{\sigma}{k\left[(\varsigma / v)^{-k}-1\right]}, & \varsigma<P_{\max }^{c}, \\ 0, & \varsigma \geq P_{\max }^{c},\end{cases}
$$

available transmission duration is

$T^{a}$

$$
= \begin{cases}\frac{\sigma}{k}\left[\left(\left(1+\frac{k x^{\mathrm{opt}}}{\sigma}\right)^{-1 / k}-\frac{\varsigma}{v}\right)^{-k}-1\right]-x^{\mathrm{opt},}, & \varsigma<P_{\max }^{c}, \\ \infty, & \varsigma \geq P_{\max }^{c},\end{cases}
$$

and maximum spectrum holes utilization is

$$
\eta_{\mathrm{SH}, \max }= \begin{cases}\left(\frac{\sigma v}{\varsigma}\right)^{(1-k) / k}, & \varsigma<P_{\max }^{c}, \\ 1, & \varsigma \geq P_{\max }^{c} .\end{cases}
$$

Proof. See Appendix D.

We can see from Corollary 4 that the optimal OSA approach is that the secondary user starts transmission at

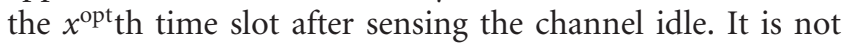
intuitive that the secondary user waits for $x^{\text {opt }}$ time slots before starting transmission after sensing the channel idle.

However, in fact, because of the long-tailed characteristic of generalized Pareto distribution, the idle period ends with greater probability at the former time slot of idle period and with less probability at the subsequent time slots. Naturally, to satisfy the constraint of collision tolerable level, the secondary user should keep away from the beginning duration of idle period, which may result in collision with more probability.

Corollary 4 also proves that collision probability of the optimal OSA approach is less than that of the following approach, where the secondary user immediately starts transmission after sensing the channel idle. Thus the optimal OSA approach is reasonable. On the other hand, we will also verify the result of Corollary 4 by simulation in the next section (see Section 6.2 ).

\section{Numerical and Simulation Results}

Our last work [15] has evaluated and verified the optimal approach in the case that the idle period is exponential distribution. Therefore, in this section, we only evaluate these cases that the idle period follows uniform distribution or generalized Pareto distribution and present numerical and simulation results to evaluate and compare the performance of the optimal OSA approach and fixed STR approach. On the other hand, it is difficult to deduce a precise expression of spectrum holes utilization in the case that the channel sensing is imperfect. Thus, we will also analyze the impact of sensing errors on optimal OSA approach by simulation.

In order to verify our conclusions, we study the performances of two approaches: transmit-first OSA (TFOSA) approach and transmit-last OSA (TLOSA) approach. TFOSA means that the secondary user starts to transmit at the first time slot and keeps transmitting for $T^{a}$, and TLOSA means that the secondary user starts to transmit at the $x_{\max }^{\text {opt }}$ th time slot and keeps transmitting until collision occurs. But they both follow the constraint of collision tolerable level.

6.1. Uniform Distribution. In this section, we study and compare the performances of the optimal OSA approach and the fixed STR approach in the case that the idle period is uniform distribution.

According to Corollary 2, the optimal OSA approach for uniform distribution is that the secondary user starts to transmit at the 1st time slot in the idle period and keeps transmitting for $T^{a}$. That is to say, the TFOSA approach is optimal OSA approach. In simulation, we generate the channel-usage patterns using uniform distribution random number generator in MATLAB and the following parameters: the expectation value of idle period $a / 2=20$ and the expectation value of busy period $1 / v=20$.

In Figure 2, we study and compare the performances of optimal OSA approach, TLOSA approach, and fixed STR approach in the case that collision tolerable level $\varsigma=0.02$. In plot (a), after the channel-usage estimate converges, spectrum holes utilization of optimal OSA approach is better than those of TLOSA approach and fixed STR approach with $D: T=4: 1$ or $D: T=1: 1$ and it converges to its theoretical value (TV of OOSA). In plot (b), after the channel-usage estimate converges, collision probability of optimal OSA approach is close to TLOSA approach, and it is greater than that of fixed STR approach with $D: T=4: 1$, but it is less than that of fixed STR approach with $D: T=1$ : $1, D: T=1: 4$, or $D: T=1: 8$, and it converges to collision tolerable level $\varsigma=0.02$.

In Figure 3, we study and compare the performances of optimal OSA approach, TLOSA approach, and fixed STR approach in the case that collision tolerable level $\varsigma=0.04$. In plot (a), after the channel-usage estimate converges, spectrum holes utilization of optimal OSA approach is better than those of TLOSA approach and all fixed STR approaches and it converges to its theoretical value (TV of OOSA). In plot (b), after the channel-usage estimate converges, collision probability of optimal OSA approach is close to TLOSA approach, and it is greater than that of fixed STR approach with $D: T=4: 1, D: T=1: 1$, or $D: T=1: 4$, but it is less than that of fixed STR approach with $D: T=1: 8$, and it converges to collision tolerable level $\varsigma=0.04$. 
From Figures 2 and 3, we can obtain the following results.

(1) The spectrum holes utilization of optimal OSA approach is much better than that of TLOSA approach and converges to its theoretical value. The collision probability of optimal OSA approach is close to that of TLOSA approach, and they are less than and converge to collision tolerable level.

(2) If the spectrum holes utilization of optimal OSA is close to that of one fixed STR approach, then the collision probability of optimal OSA approach must be much less than that of this fixed STR approach.

(3) If the collision probability of optimal OSA is close to that of one fixed STR approach, then the spectrum holes utilization of optimal OSA approach must be much greater than that of this fixed STR approach.

These results are identical to theoretical results. Thus, in the case that the idle period is uniform distribution, optimal OSA approach can adapt its access scheme according to collision tolerable level of primary user, and this approach can maximize spectrum holes utilization under collision probability constraint.

6.2. Generalized Pareto Distribution. In this section, we study and compare the performances of optimal OSA approach and fixed STR approach in the case that the idle period is Pareto distribution or generalized Pareto distribution.

According to Corollary 4, the optimal OSA approach for Pareto distribution is that the secondary user starts to transmit at the $x^{\text {opt }}$ th time slot in the idle period and keeps transmitting until collision occurs. That is, the TLOSA approach is optimal OSA approach. In simulation, we generate the channel-usage patterns using generalized Pareto distribution random number generator in MATLAB and the following parameters: the shape parameter $k=0.5$ and the scale parameter $\sigma=20$, the expectation value of busy period $1 / v=20$

In Figure 4, we study and compare the performances of optimal OSA approach, TFOSA approach, and fixed STR approach in the case that collision tolerable level $\varsigma=0.02$. In plot (a), after the channel-usage estimate converges, spectrum holes utilization of optimal OSA approach is better than those of TFOSA approach and fixed STR approach with $D: T=4: 1$ or $D: T=1: 1$ and it converges to its theoretical value (TV of OOSA). In plot (b), after the channel-usage estimate converges, collision probability of optimal OSA approach is close to that of TFOSA approach, and it is greater than that of fixed STR approach with $D: T=$ $4: 1$, but it is much less than that of fixed STR approach with $D: T=1: 1, D: T=1: 4$, or $D: T=1: 8$, and it converges to collision tolerable level $\varsigma=0.02$.

In Figure 5, we study and compare the performances of optimal OSA approach, TFOSA approach, and fixed STR approach in the case that collision tolerable level $\varsigma=0.04$. In plot (a), after the channel-usage estimate converges, spectrum holes utilization of optimal OSA approach is better than those of TFOSA approach and fixed STR approach with
$D: T=4: 1$ or $D: T=1: 1$, and it is close to that of fixed STR approach with $D: T=1: 4$, and it converges to its theoretical value. In plot (b), after the channel-usage estimate converges, collision probability of optimal OSA approach is close to those of TFOSA approach and fixed STR approach with $D: T=1: 4$, and it is greater than that of fixed STR approach with $D: T=4: 1$ or $D: T=1: 1$, but it is less than that of the fixed STR approach with $D: T=1: 8$, and it also converges to collision tolerable level $\varsigma=0.04$.

From Figures 4 and 5, we can obtain the following results.

(1) The spectrum holes utilization of optimal OSA approach is much better than that of TFOSA approach and converges to its theoretical value. The collision probability of optimal OSA is close to that of TFOSA approach, and they are less than and converge to collision tolerable level.

(2) If the spectrum holes utilization of optimal OSA is close to that of one fixed STR approach, then the collision probability of optimal OSA approach must be much less than that of this fixed STR approach.

(3) If the collision probability of optimal OSA is close to that of one fixed STR approach, then the spectrum holes utilization of optimal OSA approach must be much greater than that of this fixed STR approach.

These results are identical to theoretical results. Thus, in the case that the idle period is Pareto distribution or generalized Pareto distribution, optimal OSA approach can optimize its access scheme according to collision tolerable level of primary user, and this approach can maximize spectrum holes utilization under collision probability constraint.

6.3. Impact of Sensing Errors. In this section, we analyze the impact of sensing errors by simulation. Without loss of generality, we evaluate the impact of sensing errors on optimal OSA approach in the case that the idle period is generalized Pareto distribution. And similarly, we can also analyze this impact in the other cases. The collision tolerable level is $\varsigma=0.04$, and other settings of this simulation are the same as in the previous section.

In Figure 6, we analyze the impact of sensing errors in the case that the probability of sensing errors is 0.02 . In plot (a), after the channel-usage estimate converges, both spectrum holes utilization degradation of optimal OSA approach and that of TFOSA approach caused by sensing errors are about $2 \%$, and spectrum holes utilization of optimal OSA approach is still better than that of TFOSA approach. In (b), both collision probability increase of optimal OSA approach and that of TFOSA approach are more than 0.01 but less than 0.02 , and though collision probabilities of the two approaches are still close, they both exceed the collision tolerable level. This is problematic when the collision tolerable level is restrictive. One way to solve this problem is to set smaller collision tolerable level.

In Figure 7, we analyze the impact of different sensing errors on optimal OSA approach and TFOSA approach. In plot (a), both spectrum holes utilization of optimal OSA 
approach and that of TFOSA approach decrease almost linearly with the increase of the probability of sensing errors. In plot (b), both collision probability of optimal OSA approach and that of TFOSA approach increase by less than the probability of sensing errors. Though collision probabilities of the two approaches are still close, they both exceed the collision tolerable level. When the collision tolerable level is restrictive, it is necessary to set smaller collision constraint.

\section{Conclusion}

In this work, we consider a cognitive radio system with one primary channel and one secondary user, and then we introduce a channel-usage pattern model and a fundamental access scheme in this system. Based on this model and fundamental access scheme, we study optimal opportunistic spectrum access problem and formulate it as an optimization problem that the secondary user maximizes spectrum holes utilization under the constraint of collision tolerable level. And then we propose a unified approach to solve this optimization problem. According to the solution of the optimization problem, we analyze and present optimal opportunistic spectrum access algorithms in several cases that the idle period follows uniform distribution, exponential distribution, and Pareto or generalized Pareto distribution. Theoretical analysis and simulation results both show that the unified approach to optimal opportunistic spectrum access can maximize spectrum holes utilization under the constraint that collision probability is bounded below collision tolerable level. At last, we analyze the impact of sensing errors on spectrum holes utilization and collision probability by simulation.

In this work, we present the unified approach to optimal opportunistic spectrum access in the case that $g(x)$ is constant or monotonic function. But the optimal approach cannot solve optimal opportunistic spectrum access problem in other cases. In our future work, we will study other cases that the idle period follows any other general distribution while the busy period is general distribution.

In this work, though we only consider one secondary user, it is easily seen that the proposed approach is still effective in the case that multiple secondary users cooperate to access the idle channel. But the proposed approach is ineffective in the case that multiple secondary users compete for the idle channel. Studying the latter case will be one of the future important works.

\section{Appendices}

\section{A. Proof of Theorem 1}

Equation (14) is always true in the case that $\varsigma \geq P_{\max }^{c}$. Thus, according to (14), we can obtain that

$$
\eta_{\mathrm{SH}, \max }=\frac{E(0, \infty)}{E(0, \infty)}=1 .
$$

Therefore, we only analyze Theorem 1 in the case that $\varsigma<$ $P_{\max }^{c}$.
According to (13), we can obtain

$$
P^{C}=P_{\max }^{C} \int_{x}^{x+T} f(t) d t \leq \varsigma .
$$

According to (A.2), we can obtain that $T$ is maximal value $T^{a}$ when $P^{C}=\varsigma$, that is,

$$
\int_{x}^{x+T^{a}} f(t) d t=\frac{\varsigma}{v}
$$

We compute partial derivative of both sides of (A.3) and can obtain

$$
\left(\frac{d T^{a}}{d x}+1\right) f\left(x+T^{a}\right)=f(x) .
$$

Then, we compute partial derivative of transmission duration expectation with respect to $x$ as follows:

$$
\begin{aligned}
\frac{\partial E\left(x, T^{a}\right)}{\partial x}= & \frac{\partial}{\partial x}\left[\int_{x}^{x+T^{a}} t f(t) d t-x \int_{x}^{x+T^{a}} f(t) d t\right. \\
& \left.+T^{a} \int_{x+T^{a}}^{\infty} f(t) d t\right] \\
= & \left(1+\frac{d T^{a}}{d x}\right)\left(x+T^{a}\right) f\left(x+T^{a}\right)-x f(x) \\
& -\int_{x}^{x+T^{a}} f(t) d t \\
& -x\left[\left(1+\frac{d T^{a}}{d x}\right) f\left(x+T^{a}\right)-f(x)\right] \\
& +\frac{d T^{a}}{d x} \int_{x+T}^{\infty} f(t) d t-T^{a}\left(1+\frac{d T^{a}}{d x}\right) f\left(x+T^{a}\right) \\
= & \frac{d T^{a}}{d x} \int_{x+T^{a}}^{\infty} f(t) d t-\int_{x}^{x+T^{a}} f(t) d t \\
= & \left(\frac{f(x)}{f\left(x+T^{a}\right)}-1\right) \int_{x+T^{a}}^{\infty} f(t) d t \\
& -\int_{x}^{x+T^{a}} f(t) d t \quad(\operatorname{see}(\mathrm{A} \cdot 4)) \\
= & \frac{f(x)}{f\left(x+T^{a}\right)} \int_{x+T^{a}}^{\infty} f(t) d t-\int_{x}^{\infty} f(t) d t \\
= & \frac{\left[g\left(x+T^{a}\right)-g(x)\right]}{f(x)} .
\end{aligned}
$$

From (A.5), we can draw the following conclusions.

(1) If $g(x)$ monotonically decreases with $x$, then $\partial E\left(x, T^{a}\right) / \partial x<0$ and $E\left(x, T^{a}\right)$ decrease with $x$. Thus, the optimal access time slot is

$$
x^{\mathrm{opt}}=0,
$$

and available transmission duration $T^{a}$ satisfies

$$
\int_{0}^{T^{a}} f(t) d t=\frac{\varsigma}{v} .
$$


(2) If $g(x)$ is constant, then $\partial E\left(x, T^{a}\right) / \partial x=0$ and $E\left(x, T^{a}\right)$ are constant. Thus, the optimal access time slot is

$$
x^{\mathrm{opt}}= \begin{cases}\operatorname{arbitrary} \text { in }\left[0, x_{\max }^{\mathrm{opt}}\right], & \varsigma<P_{\max }^{c}, \\ 0, & \varsigma \geq P_{\max }^{c},\end{cases}
$$

where $x_{\max }^{\mathrm{opt}}$ satisfies

$$
\int_{x_{\max }^{\mathrm{opt}}}^{\infty} f(t) d t=\frac{\varsigma}{v}
$$

and available transmission duration $T^{a}$ satisfies

$$
\int_{x^{\mathrm{opt}}}^{x^{\mathrm{opt}}+T^{a}} f(t) d t=\frac{\varsigma}{v} .
$$

(3) If $g(x)$ is monotonically increasing with $x$, then $\partial E\left(x, T^{a}\right) / \partial x>0$ and $E\left(x, T^{a}\right)$ increase with $x$. Thus, the optimal access time slot is

$$
x^{\mathrm{opt}}= \begin{cases}x_{\max }^{\mathrm{opt}}, & \varsigma<P_{\max }^{c}, \\ 0, & \varsigma \geq P_{\max }^{c},\end{cases}
$$

where $x_{\max }^{\mathrm{opt}}$ satisfies

$$
\int_{x_{\max }^{\mathrm{opt}}}^{\infty} f(t) d t=\frac{\varsigma}{v}
$$

and available transmission duration is

$$
T^{a}=\infty .
$$

(4) It is easily obtained that the maximum spectrum holes utilization is

$$
\eta_{\mathrm{SH}, \max }=\frac{E(x, T)_{\max }}{E(0, \infty)}=\frac{E\left(x^{\mathrm{opt}}, T^{a}\right)}{E(0, \infty)} .
$$

\section{B. Proof of Corollary 2}

The probability density function of uniform distribution is

$$
f(x)= \begin{cases}\frac{1}{a} & \text { for } 0 \leq x \leq a \\ 0 & \text { for } x<0 \text { or } x>a .\end{cases}
$$

We analyze Corollary 2 in the case that $\varsigma<P_{\max }^{c}$. According to the uniform distribution, we have

$$
\begin{aligned}
g(x) & =\frac{\int_{x}^{\infty} f(t) d t}{f(x)} \\
& =\frac{\int_{x}^{a}(1 / a) d t}{(1 / a)} \\
& =a-x,
\end{aligned}
$$

that is, $g(x)$ monotonically increases with $x$.

According to Theorem 1, we can obtain that

$$
x^{\mathrm{opt}}=0
$$

and available transmission duration $T^{a}$ satisfies

$$
\begin{aligned}
& \int_{0}^{T^{a}} f(t) d t=\frac{\varsigma}{v} \\
& \Longrightarrow \int_{0}^{T^{a}} 1 / a d t=\frac{\varsigma}{v} \\
& \Longrightarrow T^{a}=\frac{a \varsigma}{v} .
\end{aligned}
$$

The transmission duration expectation of uniform distribution can be given as

$$
\begin{aligned}
E(x, T) & =\int_{x}^{x+T}(t-x) f(t) d t+T \int_{x+T}^{a} f(t) d t \\
& =\int_{x}^{x+T} t / a d t-x \int_{x}^{x+T} 1 / a d t+T \int_{x+T}^{a} 1 / a d t \\
& =\frac{(a-x-T / 2) T}{a} .
\end{aligned}
$$

It can easily obtained that

$$
\begin{aligned}
E(x, T)_{\max } & =E\left(0, \frac{a \varsigma}{v}\right) \\
& =\frac{[a-0-a \varsigma /(2 v)] a \varsigma}{(a v)} \\
& =\frac{a \varsigma}{v}-\frac{a \varsigma^{2}}{\left(2 v^{2}\right)} .
\end{aligned}
$$

It can be seen from (B.6) that $E(x, T)_{\max }$ is constant when the channel-usage and collision tolerable level are certain as

$$
\begin{aligned}
E(0, \infty) & =\int_{0}^{a} t f(t) d t \\
& =\int_{0}^{a} t / a d t \\
& =a / 2 .
\end{aligned}
$$

According to (B.6) and (B.7), we can obtain that the maximum spectrum holes utilization is

$$
\eta_{\mathrm{SH}, \max }=\frac{E(x, T)_{\max }}{E(0, \infty)}=\frac{2 \varsigma}{v}-\frac{\varsigma^{2}}{v^{2}} .
$$




\section{Proof of Corollary 3}

The probability density function of exponential distribution is

$$
f(t)=u e^{-u x} \quad \text { for } x \geq 0
$$

We analyze Corollary 3 in the case that $\varsigma<P_{\max }^{c}$. According to the exponential distribution, we have

$$
\begin{aligned}
g(x) & =\frac{\int_{x}^{\infty} f(t) d t}{f(x)} \\
& =\frac{\int_{x}^{\infty} u e^{-u t} d t}{u e^{-u x}} \\
& =\frac{e^{-u x}}{u e^{-u x}} \\
& =\frac{1}{u},
\end{aligned}
$$

that is, $g(x)$ is constant.

According to Theorem 1, we can obtain that

$$
\begin{aligned}
& \int_{x_{\max }^{\mathrm{opt}}}^{\infty} f(t) d t=\frac{\varsigma}{v} \\
& \Longrightarrow \int_{x_{\max }^{\mathrm{opt}}}^{\infty} u e^{-u t} d t=\frac{\varsigma}{v} \\
& \Longrightarrow e^{-u x_{\max }^{\mathrm{opt}}}=\frac{\varsigma}{v} \\
& \Longrightarrow x_{\max }^{\mathrm{opt}}=-\frac{\ln (\varsigma / v)}{u},
\end{aligned}
$$

and the optimal access time slot is

$$
x^{\mathrm{opt}}=\text { arbitrary in }\left[0,-\frac{\ln (\varsigma / v)}{u}\right]
$$

and available transmission duration $T^{a}$ satisfies

$$
\begin{aligned}
& \int_{x^{\mathrm{opt}}}^{x^{\mathrm{opt}}+T^{a}} f(t) d t=\frac{\varsigma}{v} \\
& \Longrightarrow \int_{x^{\mathrm{opt}}}^{x^{\mathrm{opt}}+T^{a}} u e^{-u t} d t=\frac{\varsigma}{v} \\
& \Longrightarrow e^{-u x^{\mathrm{opt}}}-e^{-u\left(x^{\mathrm{opt}}+T^{a}\right)}=\frac{\varsigma}{v} \\
& \Longrightarrow T^{a}=-\frac{\ln \left(e^{-u x^{\mathrm{opt}}}-\varsigma / v\right)}{u}-x^{\mathrm{opt}} .
\end{aligned}
$$

The transmission duration expectation of exponential distribution can be given as

$$
\begin{aligned}
E(x, T)= & \int_{x}^{x+T}(t-x) f(t) d t \\
& +T \int_{x+T}^{\infty} f(t) d t \\
= & \int_{x}^{x+T} u t e^{-u t} d t-x \int_{x}^{x+T} u e^{-u t} d t \\
& +T \int_{x+T}^{\infty} u e^{-u t} d t \\
= & \frac{1}{u} e^{-u x}-T e^{-u(x+T)} \\
& -\frac{1}{u} e^{-u(x+T)}+T e^{-u(x+T)} \\
= & \frac{1}{u} e^{-u x}-\frac{1}{u} e^{-u(x+T)} .
\end{aligned}
$$

It can easily be obtained that

$$
\begin{aligned}
E(x, T)_{\max } & =E\left(x^{\mathrm{opt}}, T^{a}\right) \\
& =\frac{1}{u} e^{-u x^{\mathrm{opt}}}-\frac{1}{u} e^{-u\left(x^{\mathrm{opt}}+T^{a}\right)} \\
& =\frac{\varsigma}{u v} .
\end{aligned}
$$

It can be seen from (C.7) that $E(x, T)_{\max }$ is constant when the channel-usage and collision tolerable level are certain as

$$
\begin{aligned}
E(0, \infty) & =\int_{0}^{\infty} t f(t) d t \\
& =\int_{0}^{\infty} u t e^{-u t} d t \\
& =\frac{1}{u} .
\end{aligned}
$$

According to (C.7) and (C.8), we can obtain that the maximum spectrum holes utilization is

$$
\eta_{\mathrm{SH}, \max }=\frac{E(x, T)_{\max }}{E(0, \infty)}=\frac{\varsigma}{v} .
$$




\section{Proof of Corollary 4}

The probability density function of generalized Pareto distribution is

$$
f(t ; k, \sigma)=\frac{1}{\sigma}\left(1+k \frac{t}{\sigma}\right)^{-1-1 / k} .
$$

We analyze Corollary 4 in the case that $\varsigma<P_{\max }^{c}$. According to the generalized Pareto distribution, we have

$$
\begin{aligned}
g(x) & =\int_{x}^{\infty} f(t) d t / f(x) \\
& =\int_{x}^{\infty} \frac{1}{\sigma}\left(1+k \frac{t}{\sigma}\right)^{-1-1 / k} d t /\left[\frac{1}{\sigma}\left(1+k \frac{x}{\sigma}\right)^{-1-1 / k}\right] \\
& =\left(1+k \frac{x}{\sigma}\right)^{-1 / k} /\left[\frac{1}{\sigma}\left(1+k \frac{x}{\sigma}\right)^{-1-1 / k}\right] \\
& =\frac{\sigma+k x}{\sigma^{2}}
\end{aligned}
$$

that is, $g(x)$ monotonically increases with $x$.

According to Theorem 1, we can obtain that

$$
\begin{aligned}
& \int_{x_{\max }^{\mathrm{opt}}}^{\infty} f(t) d t=\frac{\varsigma}{v} \\
& \Longrightarrow \int_{x_{\max }^{\mathrm{opt}}}^{\infty} \frac{1}{\sigma}\left(1+k \frac{x}{\sigma}\right)^{-1-1 / k} d t=\frac{\varsigma}{v} \\
& \Longrightarrow\left(1+k \frac{x_{\max }^{\mathrm{opt}}}{\sigma}\right)^{-1 / k}=\frac{\varsigma}{v} \\
& \Longrightarrow x_{\max }^{\mathrm{opt}}=\frac{\left[(\varsigma / v)^{-k}-1\right] \sigma}{k},
\end{aligned}
$$

the optimal access time slot is

$$
x^{\mathrm{opt}}=\frac{\left[(\varsigma / v)^{-k}-1\right] \sigma}{k},
$$

and available transmission duration is

$$
T^{a}=\infty .
$$

The transmission duration expectation of generalized Pareto distribution can be given as

$$
\begin{aligned}
& E(x, \infty)=\int_{x}^{\infty} \frac{t}{\sigma}\left(1+\frac{k t}{\sigma}\right)^{-1-1 / k} d t \\
& -x \int_{x}^{\infty} \frac{1}{\sigma}\left(1+\frac{k t}{\sigma}\right)^{-1-1 / k} d t \\
& =x\left(1+\frac{k x}{\sigma}\right)^{-1 / k} \\
& -\frac{\sigma}{k-1}\left(1+\frac{k x}{\sigma}\right)^{1-1 / k} \\
& -x\left(1+\frac{k x}{\sigma}\right)^{-1 / k} \\
& =\frac{\sigma}{1-k}\left(1+\frac{k x}{\sigma}\right)^{1-1 / k}, \\
& E(x, T)_{\max }=E\left(x^{\mathrm{opt}}, \infty\right) \\
& =\frac{\sigma}{1-k}\left(1+\frac{k\left[(\varsigma / v)^{-k}-1\right] \sigma / k}{\sigma}\right)^{1-1 / k} \\
& =\frac{\sigma}{1-k}\left(\frac{\varsigma}{v}\right)^{1-k}, \\
& E(0, \infty)=\int_{0}^{\infty} t f(t) d t \\
& =\int_{0}^{\infty} \frac{t}{\sigma}\left(1+k \frac{t}{\sigma}\right)^{-1-1 / k} d t \\
& =\frac{\sigma}{1-k} \quad(0 \leq k<1) \text {. }
\end{aligned}
$$

According to (D.7) and (D.8), we can obtain that the maximum spectrum holes utilization is

$$
\eta_{\mathrm{SH}, \max }=\frac{E(x, T)_{\max }}{E(0, \infty)}=\left(\frac{\varsigma}{v}\right)^{1-k} .
$$

\section{Acknowledgments}

This work is partially supported by the National Basic Research Program of China (no. 2007CB310608), the National High Technology Research and Development (863) Program of China (no. 2007AA01Z2B6 and no. 2007AA01Z479), the National Natural Science Foundation of China (no. 90204001), and Tsinghua-Fujitsu Joint Research Program.

\section{References}

[1] "United State Frequency Allocation Chart," http://www.ntia .doc.gov/osmhome/allochrt.pdf.

[2] SPTF, "Report of the Spectrum Efficiency Working Group," November 2002.

[3] M. McHenry, "Spectrum white space measurements," presented to New America Foundation Broadband Forum; 
Measurements by Shared Spectrum Company, June 2003, http://www.newamerica.net/files/nafmigration/archive/Doc_ File_185_1.pdf.

[4] "DARPA: The Next Generation (XG) Program," http://www .darpa.mil/sto/smallunitops/xg.html.

[5] P. J. Kolodzy, "Interference temperature: a metric for dynamic spectrum utilization," International Journal of Network Management, vol. 16, no. 2, pp. 103-113, 2006.

[6] A. Sahai, N. Hoven, and R. Tandra, "Some fundamental limits on cognitive radio," in Proceedings of the 42nd Allerton Conference on Communication, Control and Computing, Monticello, Ill, USA, October 2004.

[7] G. Ganesan and Y. G. Li, "Cooperative spectrum sensing in cognitive radio networks," in Proceedings of the 1st IEEE International Symposium on New Frontiers in Dynamic Spectrum Access Networks (DySPAN '05), pp. 137-143, Baltimore, Md, USA, November 2005.

[8] A. Ghasemi and E.S. Sousa, "Collaborative spectrum sensing for opportunistic access in fading environments," in Proceedings of the 1st IEEE International Symposium on New Frontiers in Dynamic Spectrum Access Networks (DySPAN '05), pp. 131136, Baltimore, Md, USA, November 2005.

[9] D. Cabric, S. M. Mishra, and R. W. Brodersen, "Implementation issues in spectrum sensing for cognitive radios," in Proceedings of the 38th Asilomar Conference on Signals, Systems and Computers, vol. 1, pp. 772-776, 2004.

[10] A. Fehske, J. Gaeddert, and J. H. Reed, "A new approach to signal classification using spectral correlation and neural networks," in Proceedings of the 1st IEEE International Symposium on New Frontiers in Dynamic Spectrum Access Networks (DySPAN '05), pp. 144-150, Baltimore, Md, USA, November 2005.

[11] P. A. K. Acharya, S. Singh, and H. Zheng, "Reliable open spectrum communications through proactive spectrum access," in Proceedings of the 1st International Workshop on Technology and Policy for Accessing Spectrum (TAPAS '06), Boston, Mass, USA, 2006.

[12] Y. Lei, L. Cao, and H. Zheng, "Proactive channel access in dynamic spectrum networks," in Proceedings of 2nd International Conference on Cognitive Radio Oriented Wireless Networks and Communications (CROWNCOM '07), Orlando, Fla, USA, August 2007.

[13] P. Wang, L. Xiao, S. Zhou, and J. Wang, "Optimization of detection time for channel efficiency in cognitive radio systems," in Proceedings of Wireless Communications and Networking Conference (WCNC'07), pp. 111-115, Hong Kong, March 2007.

[14] Y.-C. Liang, Y. Zeng, E. C. Y. Peh, and A. T. Hoang, "Sensingthroughput tradeoff for cognitive radio networks," IEEE Transactions on Wireless Communications, vol. 7, no. 4, pp. 1326-1337, 2008.

[15] Q. Xiao, Q. Gao, L. Xiao, S. Zhou, and J. Wang, "An optimal opportunistic spectrum access approach," in Proceedings of IEEE International Conference on Communications Workshops (ICC '09), pp. 1-5, Dresden, Germany, 2009.

[16] K. Hyoil and K. G. Shin, "Efficient discovery of spectrum opportunities with MAC-layer sensing in cognitive radio networks," IEEE Transactions on Mobile Computing, vol. 7, no. 5, pp. 533-545, 2008.

[17] S. Haykin, "Cognitive radio: brain-empowered wireless communications," IEEE Journal on Selected Areas in Communications, vol. 23, no. 2, pp. 201-220, 2005.

[18] Y. Pei, A. T. Hoang, and Y.-C. Liang, "Sensing-throughput tradeoff in cognitive radio networks: how frequently should spectrum sensing be carried out?" in Proceedings of IEEE International Symposium on Personal, Indoor and Mobile Radio Communications (PIMRC '07), April 2007.

[19] S. Geirhofer, L. Tong, and B. M. Sadler, "A measurement-based model for dynamic spectrum access in WLAN channels," in Proceedings of IEEE Military Communications Conference (MILCOM '07), pp. 1-7, Washington, DC, USA, October 2007.

[20] S. Kotz and S. Nadarajah, Extreme Value Distributions: Theory and Appilication, Imperial College Press, London, UK, 2000. 